\title{
From Authority- to Evidence-Based Medicine: Are Clini- cal Practice Guidelines Moving us Forward or Backward?
}

\author{
Michael LeFevre, MD, MSPH \\ Department of Family and Community Medicine, University of Missouri, Columbia, Missouri \\ Ann Fam Med 2017;15:410-412. https://doi.org/10.1370/afm.2141.
}

$\mathrm{O}$ ur goals in medicine should be simple; the work we do should relieve suffering, prevent future suffering, and/or prolong life. But the work we do to achieve these goals is not simple. A central part of that work is making good clinical decisions, one patient and one decision at a time. These decisions are complex, best made in the context of a relationship, integrating what we know and learn about the individual as a person and as a patient with an understanding of the biopsychosocial setting in which their story unfolds. We spend our careers learning and refining this art of doctoring. But good clinical decisions require more than bedside wisdom, they require good science. What science should we bring to the (often metaphorical) bedside? Our profession's journey of bringing science to the bedside has led us from traditional authority-based medicine to the more scientifically rigorous evidence-based medicine. The exponential growth of medical knowledge has, however, required the synthesis of that knowledge into clinical practice guidelines which, when not done well, lead us right back to authority. We have insisted on a more rigorous research base to inform clinical decisions; it is now time to insist on more uniformly rigorous clinical practice guideline development.

We can be grateful for a century of progress in the science that informs clinical decisions. In the first 50 of those years the focus was almost exclusively on the basic science of disease processes, and the science informing clinical decisions came largely from the lab.

Conflicts of interest: M.L. discloses significant prior work in clinical practice guidelines, including 10 years of service on the US Preventive Services Task Force, serving as vice-chair for 3 years and chair for 1 year.

\section{CORRESPONDING AUTHOR}

Michael LeFevre, MD, MSPH

Department of Family and Community Medicine

University of Missouri

Columbia, $\mathrm{MO}$

lefevrem@health.missouri.edu
Medical authorities brought the biomedical sciences to the bedside. As noted by Jeanne Daly ${ }^{1}$ in a historical treatise on the development and evolution of evidence based medicine, in the 1970s "questions were being asked about the validity of using traditional clinical authority as the basis for clinical decision making, and there were no grounds for appeal except by reference to the very authority that was being questioned." There was recognition that traditional authority-based decisions rooted in the lab were not adequate to assure optimal outcomes. The search for a science of clinical care led to the development and evolution of clinical epidemiology, and among many important conclusions there was widespread agreement that for clinical decisions about medical interventions, experiment trumps observation. Hypotheses about medical interventions rooted in traditional biomedical science must be subjected to well-conducted randomized controlled trials if we are to make the best decisions about the use of these interventions in our patients. This evolving science of clinical care became known as evidence-based medicine. ${ }^{2}$

But how do we empower physicians to bring evidence-based medicine to the bedside? It was perhaps from the beginning an unrealistic expectation that physicians would be aware of, read, and scientifically critique all relevant original literature and incorporate the best information into day-to-day practice. Figure 1 shows the explosion of articles indexed by MEDLINE with the key word randomized clinical trials over the last 50 years. Obviously most are not relevant to any one clinician, but the synthesis and summarization of the relevant literature informing specific clinical decisions was and remains a task not easily accomplished on top of day-to-day patient care.

Clinical practice guidelines provide physicians a shortcut to the answer to the question, "what science informs this specific clinical decision?" Clinical practice guidelines are not new; if one accepts a broad definition of what constitutes a clinical practice guideline then most medical textbooks could be considered guidelines. But if we truly aspire to bring evidence- 
based rather than authority-based medicine to our clinical decisions, then clinical practice guidelines must be the product of an explicit, rigorous, scientific process. Few are.

The National Academy of Medicine (NAM), formerly the Institute of Medicine (IOM), articulated a set of standards for the development of "Clinical Practice Guidelines We Can Trust"3 in 2011, starting with the definition: "Clinical practice guidelines are statements that include recommendations intended to optimize patient care. They are informed by a systematic review of evidence and an assessment of the benefits and harms of alternative care options." The first sentence reflects the broad scope of clinical practice guidelines, the second narrows the field substantially. Guidelines that we can trust are further restricted when one seeks adherence to the other criteria, including but not limited to transparency of process, management (not just disclosure) of conflict of interest, multidisciplinary guideline panels that establish foundations for and rating of the strength of the recommendations, informed by a systematic review that also meets explicit rigorous criteria. With the disclosure of having served on the US Preventive Services Task Force (USPSTF) for 10 years, I submit that the task force methodology comes close to meeting all of the aspirational standards of the NAM. Lessons learned highlight important constraints in the guideline development field: developing guidelines that can be trusted is hard work, labor inten- sive and thus expensive, and requires knowledge of evidence-based medicine and the process of rigorous guideline development. For these and other reasons, guidelines developed without some degree of public support often fall far short of the NAM standards.

Few clinical practice guidelines are produced by public entities, with most currently produced by specialty societies with marked variability in the rigor of the development process. ${ }^{4}$ It is ironic that we now find ourselves in the situation where the dramatic increase in science that should enable us to move from authority-based medicine to evidence-based medicine has reached a point where physicians are increasingly reliant on others to synthesize the science, but most "statements that include recommendations intended to optimize patient care" are developed by consensusbased guideline panels that actually move us back toward authority-based medicine.

What is the way forward? As we teach evidencebased medicine we also aspire to teach physicians to be able to discern whether a clinical practice guideline deserves our trust. The article by Shaughnessy, et a $1^{5}$ in this issue of the journal simplifies this evaluation, but it will still not always be an easy task. The guideline development methodology, notably management of conflict of interest, is often not transparent to the reader. Another solution, albeit more difficult to implement, would be would be to assure that fewer bad guidelines and more good guidelines are produced.

Figure 1. Number of articles referenced on MEDLINE with 'randomized controlled trial' as key word.

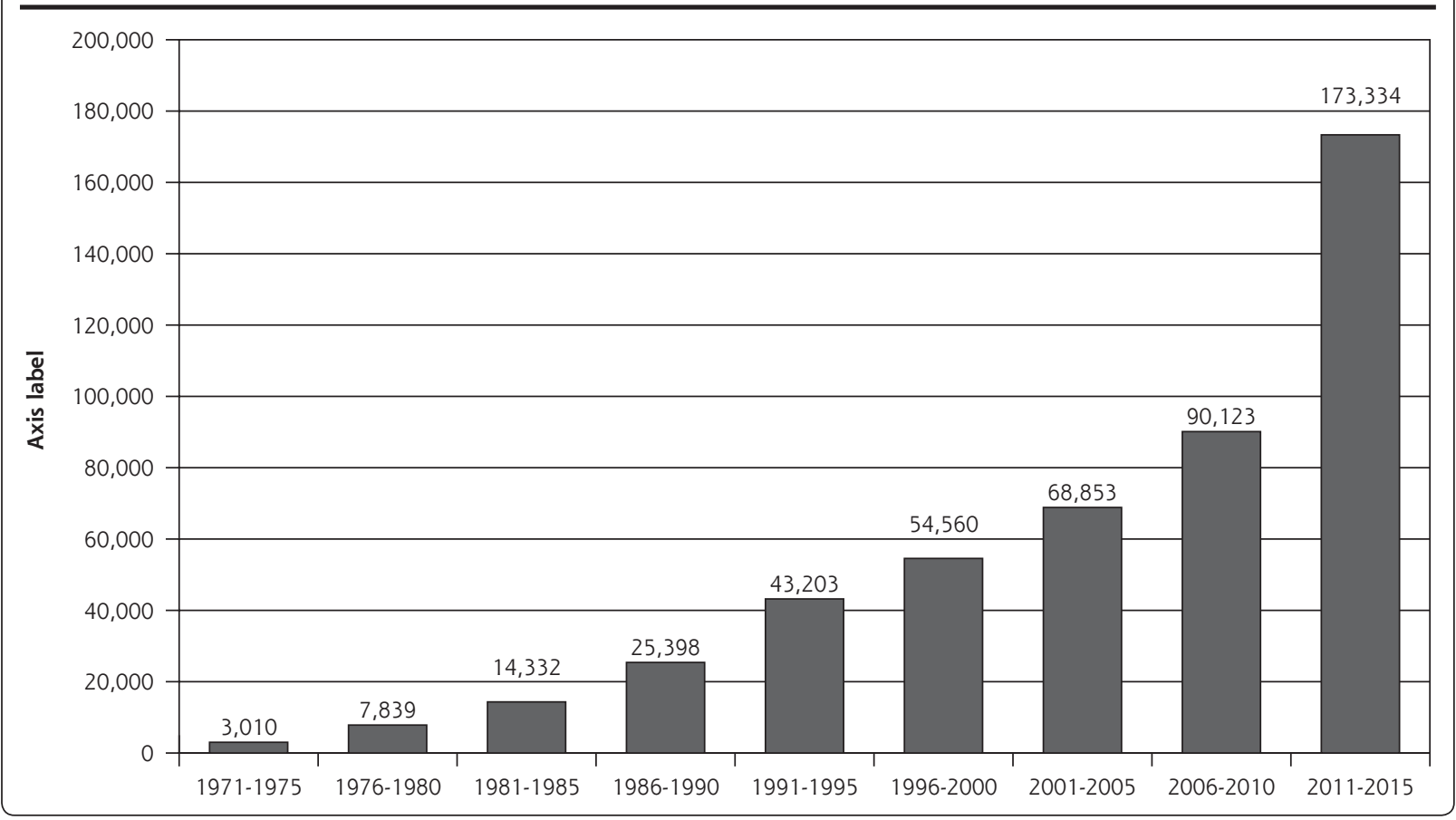


This will require a recognition that for medical care to optimally impact the health of our patients, the development of rigorous clinical practice guidelines that are useful to day-to-day clinical decision making is as important as the research that informs the guidelines. A substantial and consistent funding stream should be available for the development of clinical practice guidelines and should be awarded competitively through a process similar to research grant funding.

The logical place for this funding to occur is through the Agency for Healthcare Research and Quality (AHRQ). The process would essentially duplicate current support for the USPSTF except the topic, guideline development panel, and methodology would be part of a competitive grant proposal. Specialty societies and other organizations who currently develop clinical practice guidelines would be expected to compete. Many of the processes put in place by the USPSTF to insure transparency and manage conflict of interest, including the opportunity for public comment at each stage of the process, would be a required part of the grant proposal, and AHRQ experience with the management of this transparency could be exploited. Proposals receiving funding would also be assigned an evidence-based practice center (EPC) to work with the guideline development panel to provide an independent systematic review of the literature as currently occurs with the USPSTF and as the NAM standards suggest. The program would need additional funding, but the focus of the efforts would shift to be channeled to producing reviews that would be assured of being used in the development of a clinical practice guideline we can trust.

Methodologically rigorous clinical practice guidelines are necessary but not sufficient to move forward with bringing an ever-growing body of science to the clinical decisions we make. Usability and accessibility to a wide range of potential users, including patients, should evolve and improve. We need to expand efforts to involve communications experts in the process of guideline development from the beginning; a guideline poorly understood is a guideline poorly implemented.

The art of medicine must rest on a foundation of a good science of clinical care. It is essential that we recognize that the public good will not be served by assuming that as science evolves, physicians and their patients will use the best clinical science to inform their decisions. Clinical practice guidelines are an essential component of bringing that science to the bedside, and we must put into place a mechanism to assure that physicians and their patients have access to guidelines we can trust and guidelines we can use. Public investment is essential.

To read or post commentaries in response to this article, see it online at http://www.AnnFamMed.org/content/15/5/410.

Submitted July 4, 2017, submitted, revised, July 19, 2017; accepted July 20, 2017.

Key words: evidence-based medicine; practice guideline

\section{References}

1. Daly J. Evidence-based Medicine and the Search for a Science of Clinical Care. Oakland, CA: University of California Press; 2005.

2. Guyatt G, Cairns J, Churchill D, et al.; Evidence-Based Medicine Working Group. Evidence-based medicine. A new approach to teaching the practice of medicine. JAMA. 1992;268(17):2420-2425.

3. IOM (Institute of Medicine). Clinical Practice Guidelines We Can Trust. Washington, DC: The National Academies Press; 2011.

4. Classen DC, Mermel LA. Specialty society clinical practice guidelines: time for evolution or revolution? JAMA. 2015;314(9):871-872.

5. Shaughnessy AF, Vaswani A, Andrews BK, D’Amico F. Developing a clinician-friendly tool to identify useful clinical practice guidelines: G-TRUST. Ann Fam Med. 2017;15(5):413-418. 